\title{
Gas measurements as a mean for identification of Partial Discharges in XLPE HV cable insulation
}

\author{
Patrick Janus, Hans Edin, Kruphalan Tamil Selva \\ KTH Royal Institute of technology \\ Stockholm, Sweden
}

\begin{abstract}
Partial Discharges (PD) on high-voltage alternating current (HVAC) cables insulated with cross-linked polyethylene (XLPE) has a low occurrence, but consequences are usually severe since PD ultimately results in cable failures. Up until now the only efficient way to monitor HVAC cables for PD has been to install large coupling devices which are able to measure PDs directly from the power cables in order to verify if they are fault-free. These installations, usually of a temporary nature, are troublesome for several reasons like safety issues, measurement uncertainty, labor intensity etc.

For the purpose to ultimately create a system that is able to be utilized for PD Detection by means of gas analysis, which is easily applicable in on site, on-line conditions, initial experiments were performed in order to investigate basic material properties of XLPE and to investigate the performance of tin oxide $\left(\mathrm{SnO}_{2}\right)$ sensors for such an application. For this purpose a specialized test cell was developed in order to be able to investigate different conditions which can be expected in a cable insulation system.
\end{abstract}

It was found from the experiments that surface discharges are detectable by means of gas analysis and that these gases penetrate an XLPE sample. It was also demonstrated that the $\mathrm{SnO}_{2}$ based sensor system displays a good selectivity to the gases emitted by PD and remain inert towards other gases emitted from XLPE samples.

\section{Introduction}

Cables are an essential component in power systems on which the entire electrical infrastructure is based on. Therefore it will affect the society significantly if a high voltage cable fails. If the fault occurs on a higher level in the transmission system, entire towns or areas are affected by these outages [1]. Such situations are rare but their occurrence is increasing as the power system is ageing [2].
The faults connected to high voltage cables can be classified into two main categories [3]: the first is where the fault originates from the manufacturing and the second type of fault is connected to the handling of cables and additional components.

As to the faults connected to manufacturing it is hard to obtain any credible information as to the extent of these faults since manufacturers doesn't publish any facts or numbers concerning this. [3] However the types of faults that might be identified and attributed to manufacturing include scorching, contamination of the insulation materials, inner stresses in the cable [3]. As to faults caused by handling they include incorrect splicing, contamination of joints [3]

A common denominator for all these types of faults is that they will cause partial discharges within the insulation system. If these discharges are of a high enough magnitude and sufficiently frequent they will with time lead to a catastrophic failure. Therefore it is of interest to be able to monitor if there is on-going PD activity in a cable system, preferably under on-line conditions with a minimum work effort involved.

For this reason this paper investigates the possible detectability of premature gas evolution from PDs. The reason being to later utilize the basic knowledge gained through this paper in a prototype system which makes use of the principle of PD detection by means of PD specific trace gas analysis.

The initial assumption that the best choice for such a trace gas originating from partial discharges would be hydrogen gas, $\mathrm{H}_{2}$ [4]. The reasons being that it is known that PDs emit $\mathrm{H}_{2}, \mathrm{H}_{2}$ doesn't occur naturally in the atmosphere and since $\mathrm{H}_{2}$ is the smallest possible molecule it will have the best chance of penetrating an XLPE barrier. 
Therefore a measurement setup was created where the following questions were investigated:

1) Sensor sensitivity towards substances leaving the XLPE samples under normal conditions.

2) Sensors ability to detect gases due to Partial Discharges in the form of surface and cavity discharges on an XLPE sample.

3) The ability of the gases created by PD to penetrate an XLPE layer.

The results from these investigations are presented in this paper.

\section{Method}

\subsection{Test setup}

The basic principle of the test setup can be seen in fig 1 . The main components are the test cell in which XLPE samples are exposed to PD in the upper part and the gases generated during this process are then analyzed in the lower part of the test cell which is hermetically sealed from the upper part by the XLPE sample.

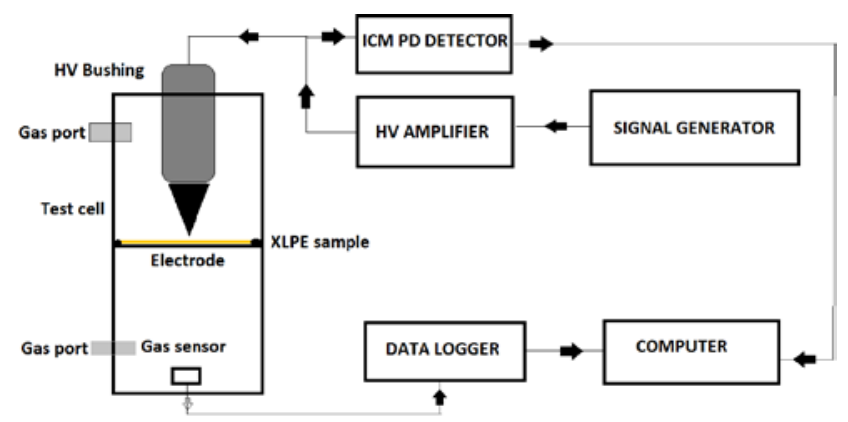

Fig. 1: Test setup

The other main components are a Trek 20/20C highvoltage amplifier which is controlled by a signal generator, a Power Diagnostix ICM system partial discharge detector which is coupled to a computer and finally the gas sensors located inside the test cell that are coupled to a computer through a Picolog 1000 series data logger.

Certain details of the set-up have been omitted for clarity since these details lies beyond the scope of this paper.

\subsection{Test cell}

For the purpose of the experiments a test cell was custom made as depicted in fig. 2. The test cell consists of a thick-walled aluminum cylinder with a sectionalizer in the middle on which the investigated polymer samples can be fixed, as can be seen in fig. 3. The sealing on both sides of the polymer sample was made gas tight so that a pressure gradient across the polymer samples could be maintained.
The validity of the pressure seal was verified every time a new XLPE sample was inserted into the test cell. This was done by evacuating all the gases in either the bottom or top part of the test cell and if the pressure increase was below 20 torr during 1 hour the test cell was deemed tight.

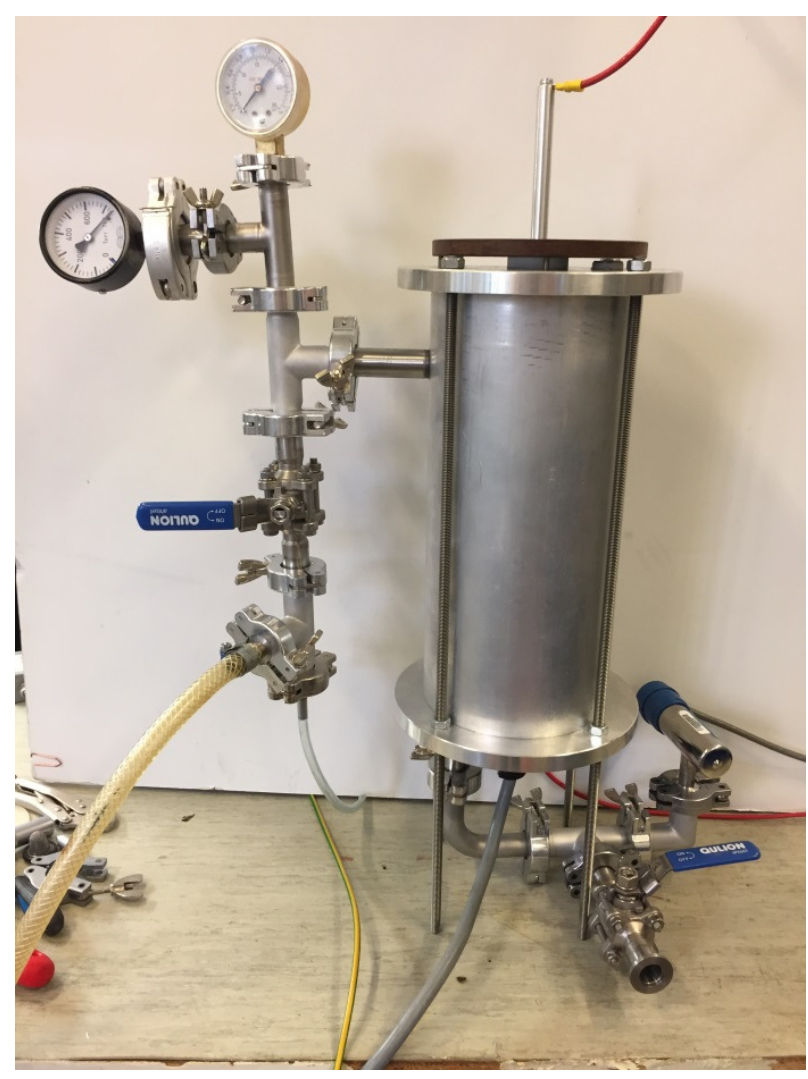

Fig 2: Fully mounted test cell with accessories

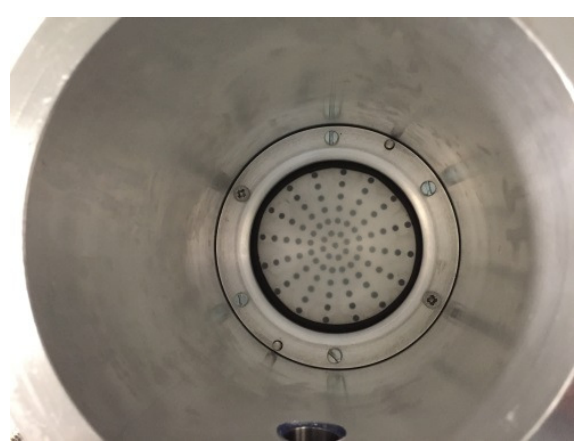

Fig. 3: XLPE sample mounted inside the test cell with a separate pressure seal gasket and aluminum sealing ring

The top part of the test cell as seen in fig. 4 consists of a circular aluminum plate with a custom made highvoltage bushing through which the electrode assembly inside of the test cell is energized. Two different types of electrode configurations were used: a needle configuration and a plate configuration as seen in fig. 4 . 


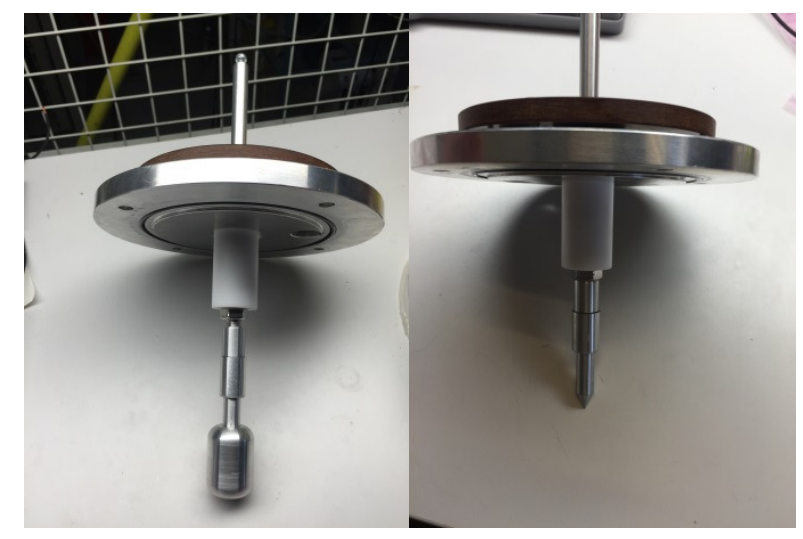

Fig. 4: Top part of test cell with flat and needle electrode mounted respectively

The bottom part of the test cell depicted in fig. 5 consists of a large circular aluminum plate and has a vacuum-tight feed-through connector for the wiring of the gas sensor printed circuit board. There is also a gas port through which gases can be either extracted or applied in the lower part of the cell.

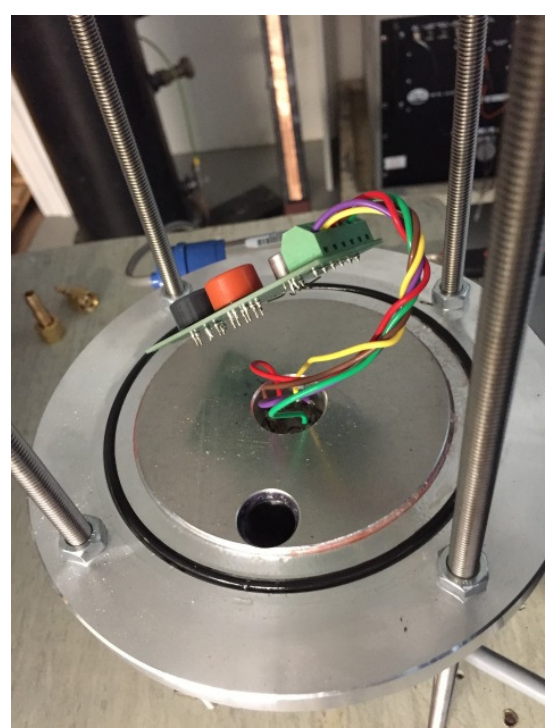

Fig. 5: Bottom part of test cell

The printed circuit board on which the gas sensors are mounted can be seen in fig. 6 contains three different sensors produced by Figaro Inc.: TGS 822, TGS 2620 and TGS 813. These are all tin oxide $\left(\mathrm{SnO}_{2}\right)$ based sensors with a broad spectrum of gases which they are sensitive to[5]. The sensing mechanism is based on detecting the resistivity changes experienced by the ntype material when surface chemisorbed oxygen reacts with reducing gases[5]. This change in resistivity is then measured by utilizing the sensor as one of the elements of a resistive voltage divider, as seen in fig. 7, where $R_{S}$ and $\mathrm{R}_{\mathrm{L}}$ form a voltage divider. In this case, $\mathrm{R}_{\mathrm{L}}$ has a fixed value of $10 \mathrm{kOhm}$. Also there is an additional heating coil (marked as $\mathrm{R}_{\mathrm{H}}$ in Fig. 7) in the sensors in order to be able to purge the sensing gases from the sensing layer of the sensors.

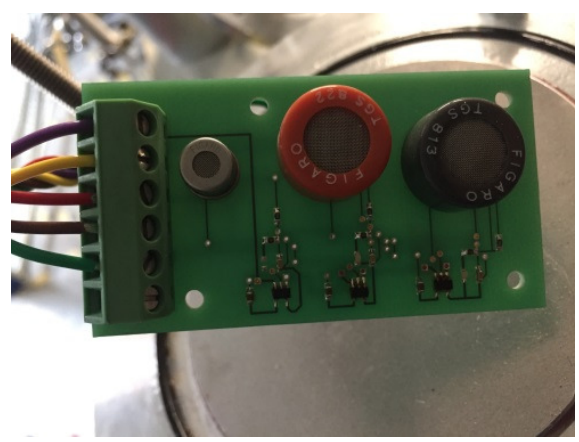

Fig. 6: Sensor printed circuit board with sensors visible

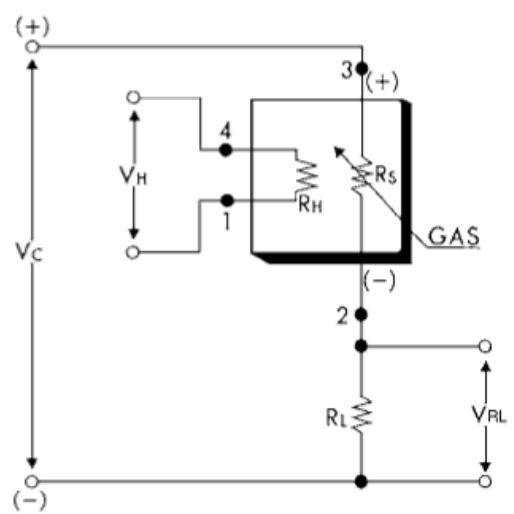

Fig. 7: Measurement circuit of the gas sensors [6]

\subsection{Measurement procedure}

After that a sample has been placed and the tightness of the test cell has been verified both sides of the test cell are drained to vacuum. When this is done the respective side is filled with a reference atmosphere consisting of dry air (as to remove the influence of different moisture content of the ambient air) up to the required level and then topped off with the required test gas.

\subsection{Sample preparation}

XLPE was provided by Borealis $\mathrm{AB}$ in the form of granules. These were pressed into samples with the aid of a polymer press with the method as described in [7]. The procedure for the polymer press was that the granules were placed between two metallic plates covered in Teflon film (to allow for good surface smoothness and easy removal of samples) with a circular aluminium spacer of $2 \mathrm{~mm}$ thickness.

The pellets were melted at $130{ }^{\circ} \mathrm{C}$ for $600 \mathrm{~s}$, followed by cross-linking at $180{ }^{\circ} \mathrm{C}$ for $1000 \mathrm{~s}$ at a pressure of 20 $\mathrm{N} / \mathrm{mm}^{2}$. The samples were the cooled down to room temperature at an approximate rate of $15^{\circ} \mathrm{C} / \mathrm{min}$.

The samples were then turned in a lathe to trim down the outer diameter to fit them precisely into the test cell. The purpose of this was to ensure that the upper and lower part of the test cell was separated by a gas-tight barrier. 


\section{Results}

\subsection{Tests with hydrogen gas}

In order to determine the ability of $\mathrm{H}_{2}$ to penetrate the XLPE samples and the sensors ability to detect the hydrogen gas an experiment was conducted where the top part of the test cell was filled with different concentrations of hydrogen: $100 \%, 10 \%$ and $1 \%$. (The remaining part was dry air filled up to ambient pressure). This procedure gave the following measurement results for the three different sensors:

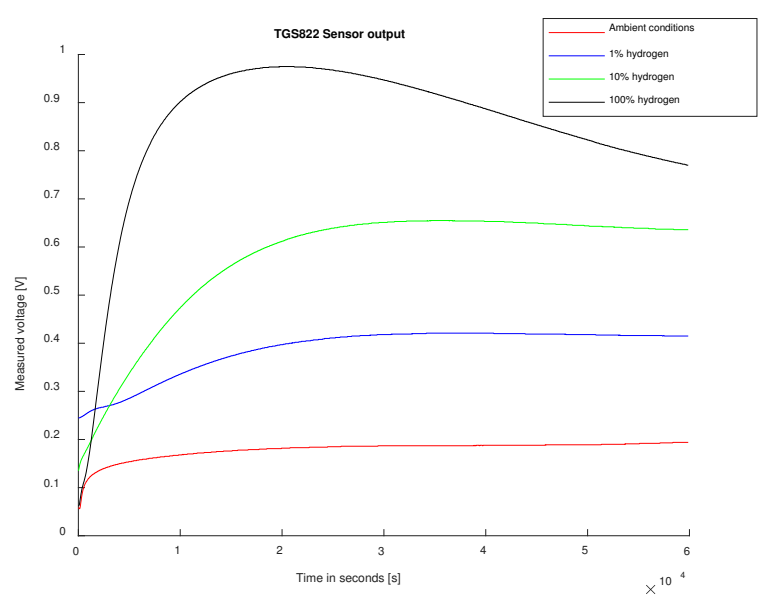

Fig. 8: TGS 822 Sensor output

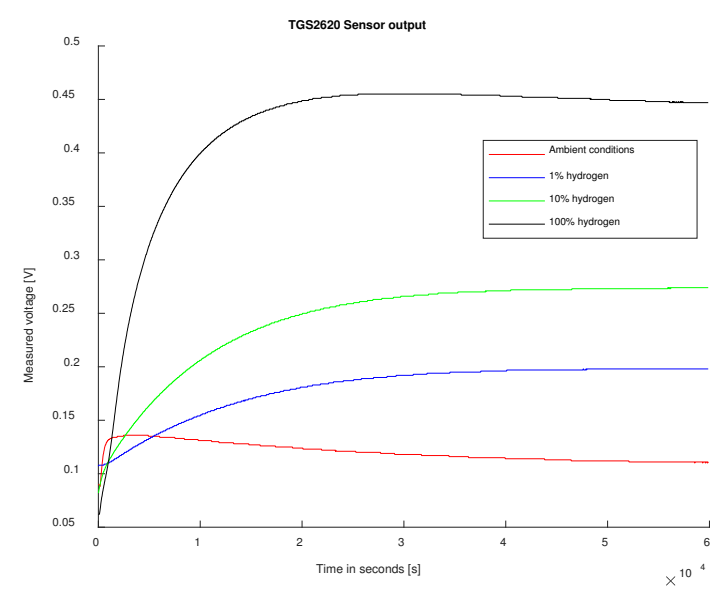

Fig. 9: TGS 2620 Sensor output

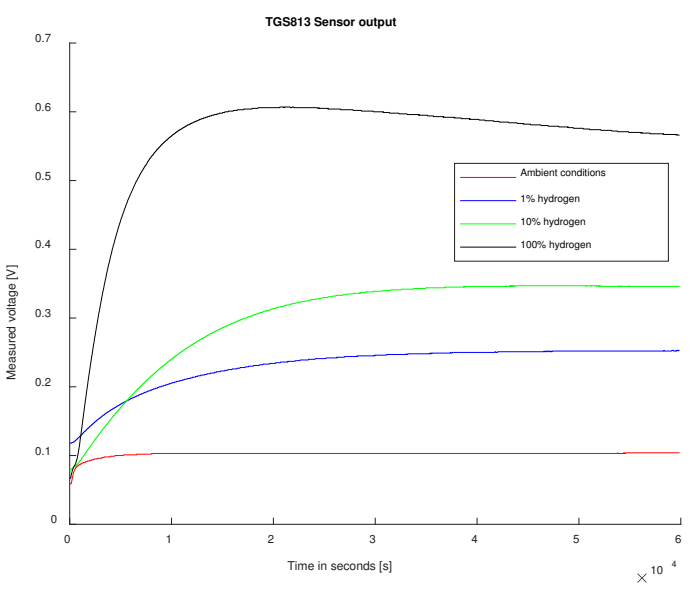

Fig. 10: TGS 813 Sensor output

What is concluded from the curves is that all sensors exhibit sensitivity towards hydrogen and that even as low concentrations as $1 \%$ are clearly detectable and distinguishable as compared to the ambient conditions where no hydrogen is present. It can also be concluded that the sensor named TGS822 seems to have the biggest dynamical range of the three sensors compared.

Another observation is that the time-constant for which the gases in the lower part of the test cell have reached concentration equilibrium is somewhere around 3 hours.

A third observation is that there seems to be an overshoot in the TGS822 sensor output with a $\mathrm{H}_{2}$ gas concentration of $100 \%$. This is, however, a phenomenon that is connected to a competition between diffusion and reaction [8] and has been described to occur in thin film sensors exposed to high concentrations of trace gases. On the other hand this gives away information on the sensors construction since the previously described process requires a thicker sensing film and since no significant overshoot can be seen for the TGS 2620 and a quite small one for TGS 813 the conclusion is drawn that the sensor with the thickest sensing film is TGS822 followed by TGS 813 and then finally comes TGS2620.

After these initial tests where the sensors sensitivity towards hydrogen has been tested and verified the following step was to initiate partial discharges over an XLPE test sample in order to see if it is possible to detect the gases caused by the partial discharges. 


\subsection{Tests with surface discharges}

Two different test scenarios were investigated where the first scenario consisted of a single layer of XLPE insulation on which PDs were initiated by a needle electrode, as can be seen in Fig. 11. The duration of the discharges was first limited to 10 minutes in order to determine if a short duration of discharges are detectable and then a continuous PD was investigated. The corresponding PD pattern can be seen in fig 12, and the sensor output is visible in fig 13. In the case of continuous PD the PD pattern in fig. 14 has had the time to settle and all contributions from initial sources have died out so it appears clearer than the pattern in fig. 12 . It also seems that there is a more constant increase in the sensor output in fig. 15 compared to fig. 13 .

One interesting observation is that the final gas concentration in the lower part of the test cell appears to be the same, no matter if the PDs have been present only for $10 \mathrm{~min}$ or for the entire duration of the measurement.

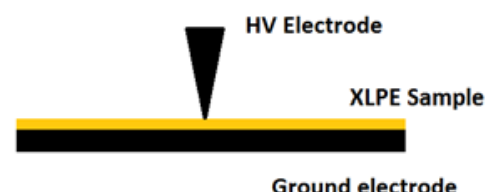

Fig 11: Test arrangement of needle-plane configuration

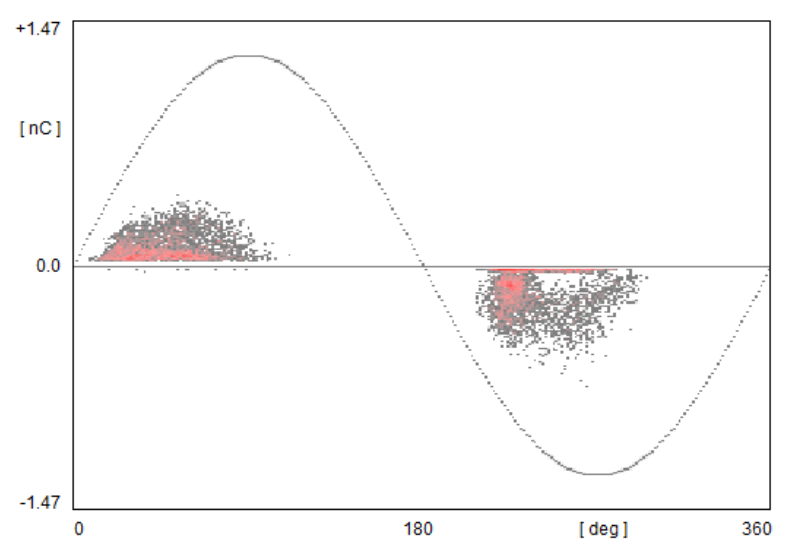

Fig. 12: PD pattern for a needle electrode on a XLPE sheet, $10 \mathrm{~min}$ of PD (PD pattern shifted $180^{\circ}$ to the phase voltage)

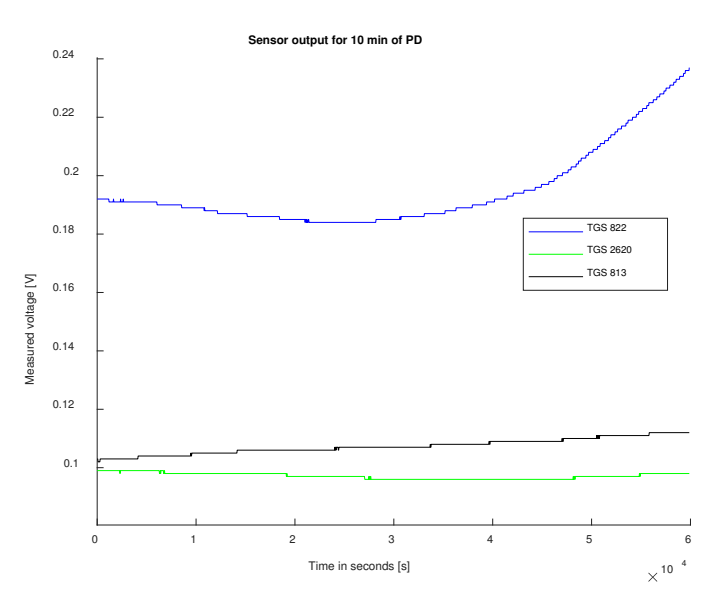

Fig 13: Sensor output for $10 \mathrm{~min}$ of PD

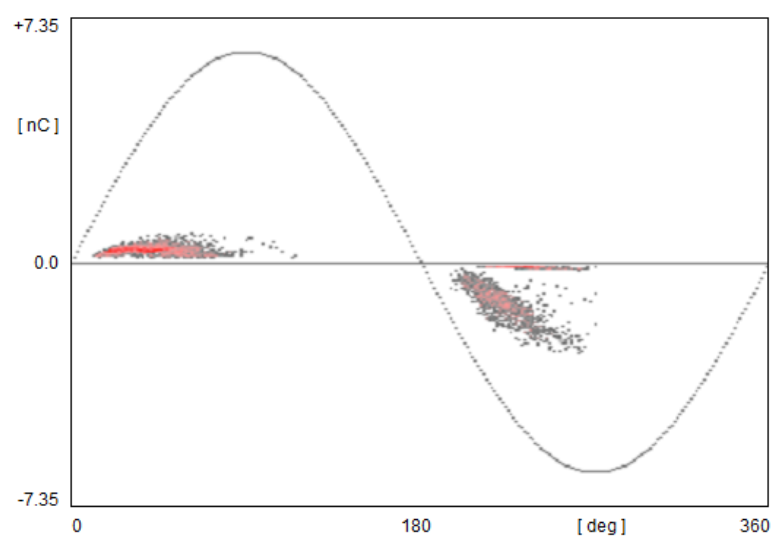

Fig. 14: PD pattern for a needle electrode on a XLPE sheet, continuous PD (PD pattern shifted $180^{\circ}$ to the phase voltage)

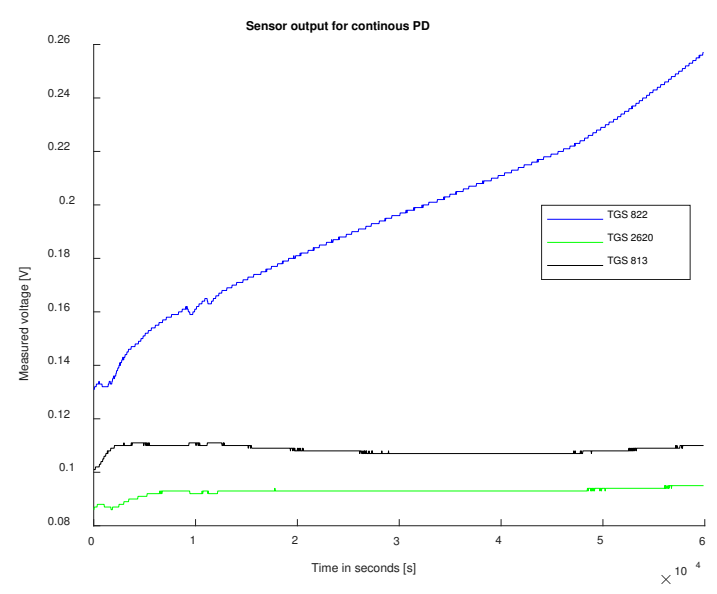

Fig 15: Sensor output for continuous PD 


\subsection{Tests with void discharges}

The second scenario comprises a cavity embedded inside XLPE insulation on which a flat electrode has been placed in order to stress the insulating material, as can be seen in Fig 16. The corresponding PD pattern is presented in fig. 17 and the sensor output is show in fig. 18. The appearance is quite similar to that of figure 15 , but the final value seems to be somewhat lower. This could probably be attributed to that the overall sample thickness is higher and that some trace gases will diffuse through the upper XLPE layer into the top part of the test cell.

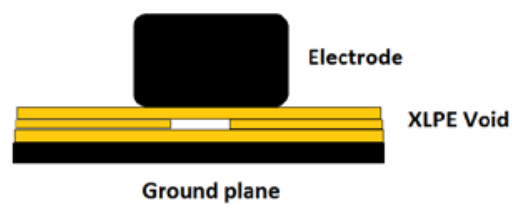

Fig 16: Test arrangement of void in XLPE

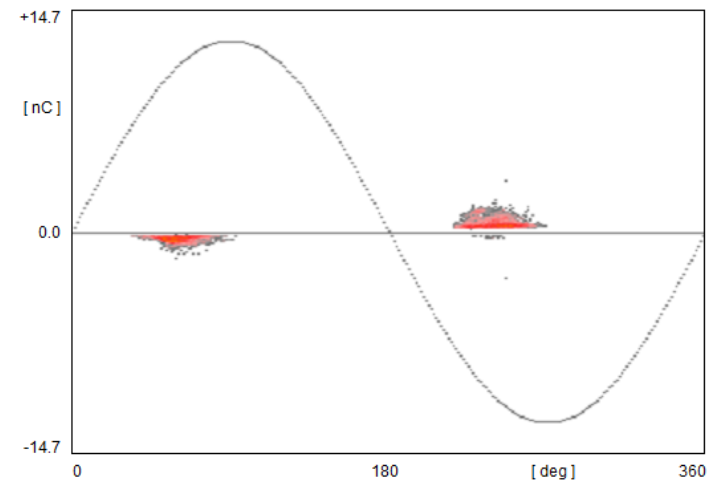

Fig. 17: PD pattern for a plate electrode on a void embedded in XLPE, continuous PD

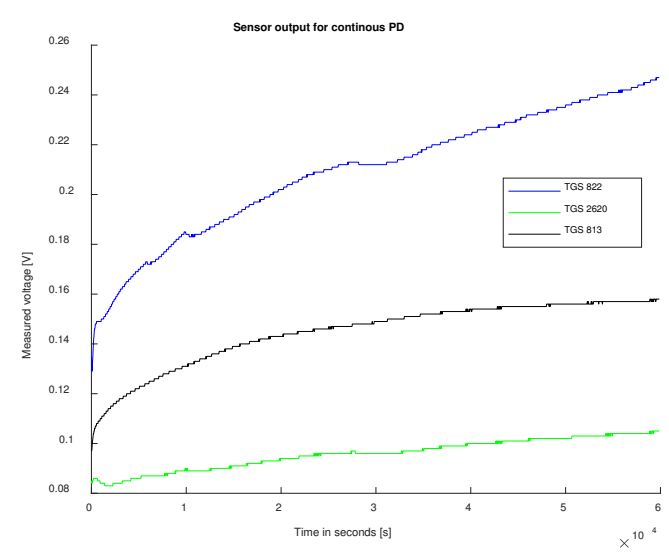

Fig 18: Sensor output for continuous PD

\section{Conclusions}

The conclusions that can be drawn are that the $\mathrm{SnO}_{2}-$ based sensors from Figaro Inc. are a viable alternative for the detection of PDs. The fact that these sensors seem to be inert towards cross-linking byproducts (like acetophenone) and gases that are emitted from the XLPE is also an encouraging fact.

It can also be concluded that sensors with a thicker sensing layer as the TGS 822 seem to be a better option since their dynamical range is much higher as compared to the other tested sensors.

It is also worth noting that the sensing system overall is very robust and hardly any interference is noted in the measurements, even that the gas sensors are placed within $10-15 \mathrm{~cm}$ of a highly energetic PD hotspot. This level of accuracy and stability is something that is usually not possible.

\section{Acknowledgements}

This work was supported by the Swegrids programme.

The authors wish to thank Borealis AB for providing the XLPE granules used as samples in this paper.

\section{References}

[1] Garcia, M., Catanach, T., Wiel, S., Bent, R., \& Lawrence, E. (2016). Line Outage Localization Using Phasor Measurement Data in Transient State. Power Systems, IEEE Transactions on, 31(4), 3019-3027.

[2] Chakravorti, S., Dey, D., Chatterjee, B., \& SpringerLink. (2013). Recent Trends in the Condition Monitoring of Transformers Theory, Implementation and Analysis (Power Systems).

[3] Yun Jiang, Hong Min, Junhua Luo, Yu Li, Hai Li, Xiaojuan Jiang, ... Wenjie Li. (2010). Partial discharge pattern Characteristic of MV Cable joints with artificial defect. Electricity Distribution (CICED), 2010 China International Conference on, 1-3.

[4]Wolter, Tanaka, \& Johnson. (1982). A Study of the Gaseous Degradation Products of Corona-Exposed Polyethylene. Electrical Insulation, IEEE Transactions on, EI-17(3), 248-252.

[5] Díaz Delgado, Raül, Morante I Lleonart, Joan Ramon, \& Sanz Carrasco, Fausto. (2002). Tin Oxide Gas Sensors: An Electrochemical Approach.

[6]Figaro Inc., 2002, Product information TGS 813 for the detection of combustible gases Available at: http://www.figarosensor.com /products/813pdf.pdf (accessed 4/3 2017)

[7] U. H. Nilsson, J. Andersson, V. Englund, V. Eriksson, P. O. Hagstrand and A. Smedberg, "The role and measurement of DC conductivity for HVDC cable insulation materials," 2015 IEEE Conference on Electrical Insulation and Dielectric Phenomena (CEIDP), Ann Arbor, MI, 2015, pp. 31-34.

[8] Matsunaga, Sakai, Shimanoe, \& Yamazoe. (2002). Diffusion equation-based study of thin film semiconductor gas sensor-response transient. Sensors \& Actuators: B. Chemical, 83(1-3), 216-221. 